\title{
A preliminary investigation of mechanisms by which short-term resistance training increases strength of partially paralysed muscles in people with spinal cord injury
}

\author{
Elizabeth A. Bye ${ }^{1,2} \cdot$ Lisa A. Harvey ${ }^{1} \cdot$ Joanne V. Glinsky ${ }^{1} \cdot$ Bart Bolsterlee $^{3,4} \cdot$ Robert D. Herbert $^{3,4}$
}

Received: 27 September 2018 / Revised: 16 April 2019 / Accepted: 23 April 2019 / Published online: 15 May 2019

(c) International Spinal Cord Society 2019

\begin{abstract}
Study design Pretest-posttest design.

Objectives To investigate mechanisms by which short-term resistance training ( 6 weeks) increases strength of partially paralysed muscles in people with spinal cord injury (SCI).

Setting Community-based setting, Sydney, Australia.

Participants Ten community-dwelling people with partial paralysis of elbow flexor, elbow extensor, knee flexor or knee extensor muscles following SCI (range 5 months to 14 years since injury).

Methods Muscle architecture and strength were assessed before and after participants underwent a six week strengthtraining program targeting one partially paralysed muscle group. The outcome of primary interest was physiological cross sectional area (PCSA) of the trained muscle group measured using magnetic resonance imaging (MRI) and diffusion tensor imaging (DTI). Other outcomes were changes in mean muscle fascicle length, muscle volume, pennation angle, isometric strength and muscle strength graded on a 13-point scale.

Results The mean increase in maximal isometric muscle strength was $14 \%$ (95\% CI, -3 to $30 \%)$ and 1.5 points (95\% CI, 0.5 to 2.5 ) on the 13-point manual muscle test. There was no evidence of a change in muscle architecture.

Conclusion This study is the first to examine the mechanisms by which voluntary strength training increases strength of partially paralysed muscles in people with SCI. The data suggest that strength gains produced by six weeks of strength training are not caused by changes in muscle architecture. This suggests short-term strength gains are due to increased neural drive or an increase in specific muscle tension.
\end{abstract}

Supplementary information The online version of this article (https:// doi.org/10.1038/s41393-019-0284-2) contains supplementary material, which is available to authorized users.

Robert D. Herbert

r.herbert@neura.edu.au

1 John Walsh Centre for Rehabilitation Research, Sydney Medical School/Northern, University of Sydney, St Leonards, NSW, Australia

2 Spinal Injury Unit, Prince of Wales Hospital, Randwick, NSW, Australia

3 Neuroscience Research Australia (NeuRA), Randwick, NSW, Australia

4 University of New South Wales, Randwick, NSW, Australia

\section{Introduction}

Partial paralysis is one of the most common impairments experienced after spinal cord injury (SCI). Partial paralysis of a specific muscle is caused by disruption to some but not all the motor pathways to that muscle [1]. The paralysis can occur at or below the level of injury. Partial paralysis manifests as weakness (a reduction in the ability to generate muscle force voluntarily), which can severely impair motor function [2].

Previous studies have demonstrated the effectiveness of strength training for improving voluntary strength in the partially paralysed muscles of people with SCI [3-5]. For example, we conducted a randomized controlled trial in which participants underwent strength training for 12 weeks [3]. Training increased strength by a mean of $4.3 \mathrm{Nm}(95 \%$ CI; 1.9 to 6.8 ) or $24 \%$ of initial strength. 
In able-bodied individuals (i.e., people without paralysis) the mechanisms that contribute to strength gains are quite well understood. Both central and peripheral factors induce gains in strength [6-8]. It is thought that neural adaptations (central factors) occur soon after strength training is initiated. Substantial peripheral adaptations (such as muscle hypertrophy) are typically not observed until after 6 or 8 weeks of strength training [9-13]. However, we hypothesised that, in people who have partial paralysis and are very weak, hypertrophy might occur earlier than 6 weeks [14].

The mechanisms by which training increases strength in partially paralysed muscles following SCI are not known. However one small randomised trial $(n=9)$ used MRI to measure muscle anatomical cross-sectional area (ACSA) of completely paralysed quadriceps muscles before and after 12 weeks of training with neuromuscular electrical stimulation against resistance [15]. Training increased the ACSA of the knee extensor muscles by $35 \%$ and increased the ACSA of the knee flexors by $16 \%$. As this study used electrically stimulated contractions to increase the strength of completely paralysed muscles it is not possible to draw conclusions from this study about the response of partially paralysed muscles to voluntary resistance training.

A key issue in quantifying peripheral responses to training is the measurement of muscle cross-sectional area. The physiological cross-sectional area (PCSA) of a muscle is the area of a hypothetical slice that passes transversely through all of the fibres of the muscle $[16,17]$. It is the main peripheral determinant of the intrinsic force generating capacity of a muscle [16]. The best way to calculate PCSA is to divide muscle volume by mean fascicle length. Usually muscle volume is measured with MRI, CT scans, or ultrasound imaging [18-20], and fascicle length is measured with ultrasound imaging [21]. A limitation of ultrasound measurements of fascicle length is that they are obtained from planar (two-dimensional) images, usually at just one site in the muscle [22-24].

New methods, based on MRI and DTI, can be used to measure muscle fascicle length of whole muscles in three dimensions [25, 26]. These methods potentially provide more accurate measures of PCSA than methods that use ultrasound measurements of muscle volume and muscle fascicle length. DTI has not been used yet to measure training-induced changes in muscle architecture in people with SCI.

We conducted a preliminary investigation of the mechanisms by which short-term voluntary strength training increases voluntary muscle strength in partially paralysed muscles following SCI. A novel feature of this study is the use of MRI and DTI to measure changes in muscle architecture, such as changes in PCSA that may contribute to changes in strength.

\section{Methods}

A single-group pretest-posttest study was conducted on community-dwelling people with chronic SCI. Participants trained a partially paralysed muscle group for 6 weeks. Muscle architecture was measured before and after training using MRI and DTI. The trial was retrospectively registered with the Australian New Zealand Clinical Trials Registry (ACTRN12618001606279). All applicable institutional and governmental regulations concerning the ethical use of human volunteers were followed. The study was approved by the relevant ethics committees. Participants gave written informed consent.

\section{Participants}

Ten people with SCI were recruited from the community in Sydney, Australia. People were eligible to participate if they had any type of SCI (i.e., complete or incomplete as defined by the International Standards for Neurological classification of SCI (ISNCSCI)), had partial paralysis (grade 1 to 4 strength on a 6-point manual muscle test [27]) in one of the target muscle groups (elbow flexor, elbow extensor, knee flexor or knee extensor muscles), were aged 18 years or over at the time of consent, were willing to participate in the trial, and did not have any neurological condition other than the spinal cord lesion.

People were excluded if they had any condition preventing testing or training of the target muscle group (for example unstable fractures in the target limb or uncontrolled spasticity), had completed more than three consecutive weeks in the last 3 months of progressive resistance training for the target muscle group, were unable to co-operate, had insufficient English to provide informed consent, or were expected to be unable to remain still in the MRI scanner for the duration of the scan.

\section{Intervention}

One target muscle group was selected for each participant from the following muscle groups: elbow flexors, elbow extensors, knee flexors or knee extensors. The selected muscle group was partially paralysed. If more than one muscle group was suitable for inclusion, we chose the muscle group expected to benefit most from strength training or the muscle group the participant most wanted to train. Participants trained the target muscle group on one side of the body, three times a week for 6 weeks. The training program adhered to the principles of progressive resistance training. Training consisted of 40 maximal contractions in four sets of ten, with 2 min rest between sets. The first two sets of ten repetitions were isometric contractions and the second two sets of ten repetitions were 
concentric contractions. We chose to train participants using concentric and isometric contractions because the findings of a small crossover trial, one of the few trials of strength training in individuals with SCI, suggested isometric training produced larger increases in strength than concentric strength training [5]. Resistance was applied manually by a therapist because, with very weak muscles, it is difficult or impossible to provide the target resistance using free weights or isokinetic dynamometry. The therapist ensured that the resistance was sufficient to exhaust the participant by the end of the set, so that the participant was unable to perform another intense contraction after the tenth. If, over the course of the training program, a participant's strength increased to the extent that the therapist was no longer able to provide sufficient resistance, weights were applied to the participant's limb to provide additional resistance. When training sessions were missed, additional sessions were provided to make up missed sessions.

\section{Assessment}

Participants had an MRI scan and underwent a strength assessment prior to commencing the strength-training program. The scan and strength assessment were repeated 48-72 $\mathrm{h}$ after the last training session. The outcome of primary interest was the PCSA of the trained muscle group. Other outcomes were mean fascicle length, muscle volume, mean pennation angle, isometric strength and muscle strength graded on a 13-point scale.

\section{Muscle architecture (PCSA, mean fascicle length, muscle volume and pennation angle)}

Participants were positioned supine on the MRI scanner bed. If scanning the hamstrings, a wedge was placed under the knee so that the thigh was suspended to avoid compression of the muscle from the weight of the leg. Scans were obtained at a single joint angle. The participant was asked to remain relaxed and still during the scan.

A 3 Tesla MRI scanner (Achieva TX; Philips Medical Systems, Best, The Netherlands) with a 32-channel cardiac coil was used to obtain mDixon, T1-weighted (Fig. 1) and DTI images of the thigh from the proximal end of the femur to the top of the knee joint or for the upper arm from the proximal end of the humerus to the elbow joint. The following imaging parameters were used. mDixon: 2-point 3D multi-echo mDixon fast field echo (FFE) sequence, TR/TE1/ $\mathrm{TE} 2=5.9 / 3.5 / 4.6 \mathrm{~ms}$, field of view $(\mathrm{FOV})=180 \times 180 \mathrm{~mm}$, acquisition matrix $=180 \times 180$ (reconstructed to $192 \times 192$ ), slice $=1 \mathrm{~mm}$, number of slices $=320$ and scan time of $334 \mathrm{~s}$. T1-weighted: TSE sequence, TR/TE $=700 / 12 \mathrm{~ms}, \mathrm{FOV}=$ $180 \times 180 \mathrm{~mm}$, acquisition matrix $=256 \times 188$ (reconstructed to $864 \times 864$ ), slice $=4 \mathrm{~mm}$, number of slices $=80$ and scan time of $250 \mathrm{~s}$. DTI: EPI sequence, TR/TE $=9050 /$ $60 \mathrm{~ms}$, diffusion gradient time $\Delta / \delta=29.6 / 8.2 \mathrm{~ms}, \mathrm{FOV}=$ $180 \times 180 \mathrm{~mm}$, acquisition matrix $=80 \times 78$ (reconstructed to $112 \times 112$ ), slice $=5 \mathrm{~mm}$, number of slices $=50$, number of signal averages $=4, b$-value $=500 \mathrm{~s} / \mathrm{mm}^{2}$ (b0 with $b=0$ $\mathrm{s} / \mathrm{mm}^{2}$ ), number of gradient directions $=16$ on a hemisphere, fat suppression: spectral attenuated inversion recovery (SPAIR) and scan time of $606 \mathrm{~s}$. To correct for local inhomogeneities in the magnetic field, the DTI scan was preceded by a B0-calibration using the following settings: 3D FFE, $\mathrm{FOV}=180 \times 180 \mathrm{~mm}$ (reconstructed to $112 \times 112$ ), slice $=$ $5 \mathrm{~mm}$, number of slices $=50, \mathrm{TR} / \mathrm{TE} / \Delta \mathrm{TE}=30 / 4.6 / 2.3 \mathrm{~ms}$, $\mathrm{NSA}=2$.Total scan time was $\sim 23$ min per session.

\section{Muscle segmentation}

The quadriceps, hamstrings, biceps or triceps muscles were manually outlined (segmented) using imaging processing software (ITK-SNAP, www.itksnap.org). We manually segmented one of the (pre-or post-training) images for each participant and then used registration algorithms (Elastix) $[28,29]$ to segment the second image based on the first. Segmentation was carried out by a researcher who did not know whether the scan was obtained before or after training.

Muscle architecture measurements were extracted from the anatomical MRI scans and DTI data using methods described in more detail elsewhere [26]. Briefly, threedimensional muscle surface models were created from the muscle segmentations. Muscle volumes were defined as the volumes of the surface models. Deterministic DTI tractography algorithms were used to generate a large number of fibre tracts within a muscle. The fibre tracts were extended so that they terminated on the muscle surface or on intramuscular tendons, like real muscle fascicles [26]. Fascicle reconstructions were only included if the angle between the slopes at either endpoint of a fascicle was between 135 and $180^{\circ}$ (so that fascicles could not curve back in on themselves), and fascicles were longer than $20 \mathrm{~mm}$ and shorter than $200 \mathrm{~mm}$, were extrapolated to the aponeuroses by $<40 \%$ of their total length, and had curvatures $<30 / \mathrm{m}$. Reconstructions of all muscles were visually inspected by a team of researchers who were blinded to whether the reconstruction was obtained before or after training. Reconstructions that showed unrealistic alignment of muscle fascicles or had a sparse distribution of fascicles were excluded from further analysis. The fascicle length and pennation angle of a muscle were calculated as the median fascicle length and pennation angle of all fascicles reconstructed in that muscle. To determine the effect of training, the mean measurement of muscle fascicle length or pennation of all muscles of all participants before training was compared to the mean value after training. PCSA was 
Fig. 1 Example of transverse slices from the a T1-weighted scan and $\mathbf{b}$ mDixon scan (water image) obtained approximately mid-thigh in one participant. c Example of a three-dimensional reconstruction of the surface and fascicles (shown as lines) of the biceps femoris longhead. The T1-weighted image is shown as well
A

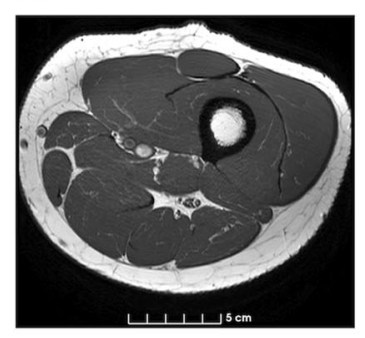

B

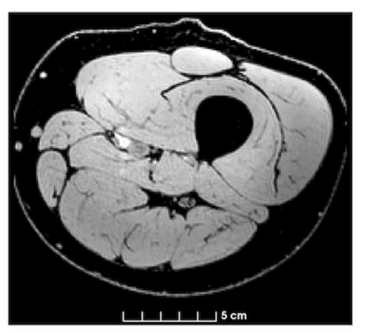

C

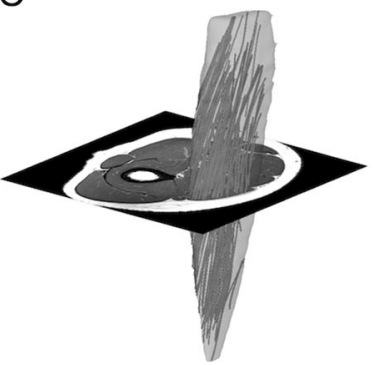

calculated by dividing muscle volume by median fascicle length.

\section{Muscle strength}

Isometric strength of the target muscle was tested using a dynamometer (Cybex Norm with Humac, CSMi, Stoughton, MA, USA). Participants were positioned in the testing chair with the target limb firmly strapped to the dynamometer arm. They were asked to perform four maximal contractions, lasting about three to five seconds each, while being provided visual feedback of the torque they produced and verbal encouragement to push as hard as they could. A one-minute rest was given between contractions. The largest force measured during the four contractions was taken to be the participant's isometric strength.

Muscle strength was also measured using a 13-point manual muscle test scale (see Table 1). This scale was adapted from the traditional 0 to 5 point manual muscle test by adding scale increments.

\section{Statistical analysis}

\section{Effects of training}

The mean effects of short-term training on PCSA, mean fascicle length, volume and pennation angle were estimated from the mean changes over the training period. Confidence intervals were obtained using the $t$-distribution.

\section{Sample size}

Sample size calculations were based on the changes in ACSA with 12 weeks of training reported by Gregory et al. [30]. They reported mean (SD) changes in the ACSA of the ankle plantarflexor and knee extensor muscles of 14\% (SD 4) and $8 \%$ (SD 2), respectively. If we conservatively assume the larger SD (4\%), a sample size of 10 participants would provide a better than $90 \%$ power to detect an increase in cross-sectional area of $5 \%$ with a two-tailed paired- samples test and a rejection probability under the null hypothesis of $5 \%$.

\section{Results}

The demographic characteristics of the 10 participants are presented in Table 2. Most participants had chronic injuries, and all had incomplete injuries with neurological levels ranging from $\mathrm{C} 3$ to $\mathrm{L} 1$ and motor levels ranging from $\mathrm{C} 3$ to L3 as defined by the ISNCSCI.

The protocol dictated that participants train three times a week for 6 weeks (total 18 training sessions). This was largely achieved: a median of 18 training sessions (IQR 17 to 18) was provided over the six weeks. No participant withdrew from the study.

On average, muscle volume increased by $1 \%(95 \% \mathrm{CI}$, -5 to $6 \%$ ), PCSA decreased by $5 \%$ (95\% CI, -16 to $6 \%$ ), mean fascicle length increased by $8 \%(95 \% \mathrm{CI},-7$ to $24 \%)$ and mean pennation did not change (mean $0 \%$; $95 \% \mathrm{CI}$, -12 to 13 ) in the trained muscle. None of these effects were statistically significant ( $p>0.05$; Fig. 2 and supplementary data).

The mean increase in maximal isometric muscle strength measured using the isokinetic dynamometer was $14 \%(95 \%$ CI, -3 to $30 \%$ ). The mean change in strength measured on the 13-point manual muscle test scale was 1.5 points $(95 \%$ CI, 0.5 to 2.5 ).

\section{Discussion}

This exploratory study constitutes a first step towards understanding the mechanisms by which short-term voluntary strength training increases the strength of partially paralysed muscles in people with spinal cord injury. To our knowledge, no previous studies have examined the effects of voluntary strength training on muscle architecture following SCI. We found that 6 weeks of strength training increased the isometric strength of partially paralysed 
Table 1 13-point manual muscle test

\begin{tabular}{lll}
\hline Grade & Points & \\
\hline 0 & 0 & No palpable contraction \\
1 & 1 & Visible or palpable contraction but unable to move \\
$1+$ & 2 & Able to move through a small range with gravity eliminated \\
$2-$ & 3 & Able to move through a large range with gravity eliminated \\
2 & 4 & Able to move through a full range with gravity eliminated \\
$2+$ & 5 & Able to move through a small range against gravity \\
$3-$ & 6 & Able to move through a large range against gravity \\
3 & 7 & Able to move through a full range against gravity \\
$3+$ & 8 & Able to move through a small range with light resistance \\
$4-$ & 9 & Able to move through a full range with light resistance \\
4 & 10 & Able to move through a full range with moderate resistance \\
$4+$ & 11 & Able to move through a full range with heavy resistance \\
5 & 12 & Normal strength
\end{tabular}

Table 2 Characteristics of participants $(n=10)$

\begin{tabular}{|c|c|c|}
\hline Age (years), median (IQR) & 42 (33 to 62$)$ & \\
\hline $\operatorname{Sex}(\mathrm{F}: \mathrm{M})$ & $3: 7$ & \\
\hline \multicolumn{3}{|l|}{ Neurological level } \\
\hline $\mathrm{C} 1-4$ & 3 & \\
\hline C5-8 & 1 & \\
\hline T1-S5 & 6 & \\
\hline \multicolumn{3}{|l|}{ Trained muscle } \\
\hline Elbow flexors & 1 & \\
\hline Elbow extensors & 0 & \\
\hline Knee flexors & 6 & \\
\hline Knee extensors & 3 & \\
\hline Time since injury (years), median (IQR) & 1.9 (1.1 to 3.9$)$ & \\
\hline \multicolumn{3}{|l|}{ AIS classification } \\
\hline A & 0 & \\
\hline $\mathrm{B}$ & 0 & \\
\hline $\mathrm{C}$ & 4 & \\
\hline $\mathrm{D}$ & 6 & \\
\hline Motor level & Left & Right \\
\hline $\mathrm{C} 1-4$ & 2 & 1 \\
\hline $\mathrm{C} 5-8$ & 2 & 3 \\
\hline T1-S5 & 6 & 6 \\
\hline
\end{tabular}

muscles by on average, $14 \%$ and by 1.5 points on the 13 point manual muscle test scale. We did not nominate a minimally worthwhile treatment effect prior to the commencement of the study because the study was primarily designed to explain the possible mechanisms underlying strength changes. Nonetheless, most would consider an increase of $14 \%$ (as measured with dynamometry) and $11 \%$ (as measured with the 13-point manual muscle test) as probably meaningful for most patients. Interestingly, despite the increase in strength we found no evidence of a systematic change in muscle architecture. The observation that strength increased but muscle architecture did not suggests that the increases in strength were mediated by mechanisms other than by changing muscle architecture (e.g., muscle hypertrophy). One such mechanism could be an increase in muscle specific tension (i.e., an increase in the intrinsic capacity of the muscle to generate force per unit of cross-sectional area.). The second possible mechanism could be by enhancement of the excitation provided by the nervous system to the muscle (neural drive). We cannot be sure how much each of these mechanisms contributed to the gains in strength as we did not measure specific tension or muscle activation.

The study had several limitations. We did not endeavour to conduct a parallel randomised controlled trial because that would have required a much larger number of scans and the cost of conducting and analyzing scans is high. We considered conducting a randomised cross-over design but an analysis suggested the potential bias associated with any achievable wash-out period would probably be greater than the bias inherent in a (single-group) pretest-posttest design. Therefore, we used a pretest-posttest design. The lack of randomisation means that we cannot completely rule out the possibility that the findings were biased by changes in muscle architecture that would have occurred over time without training, or which were an artifact of repeated measurement. However majority of participants had chronic injuries, so it is unlikely that muscle architecture would have changed over a 6 week period. A larger randomised study with a control group would provide a more rigorous test of our findings.

Another limitation to the study, which may explain the lack of change in muscle architecture, is the short training period (6 weeks). In able-bodied individuals, changes in 
Fig. 2 Pre- and post-training measurements of muscle volume, PCSA, median fascicle length, median pennation angle and strength in all 10 participants. Bars and error bars are means and SDs. Data from individual participants are shown with participant-specific symbols. KE knee extensors, KF knee flexors, EF elbow flexors
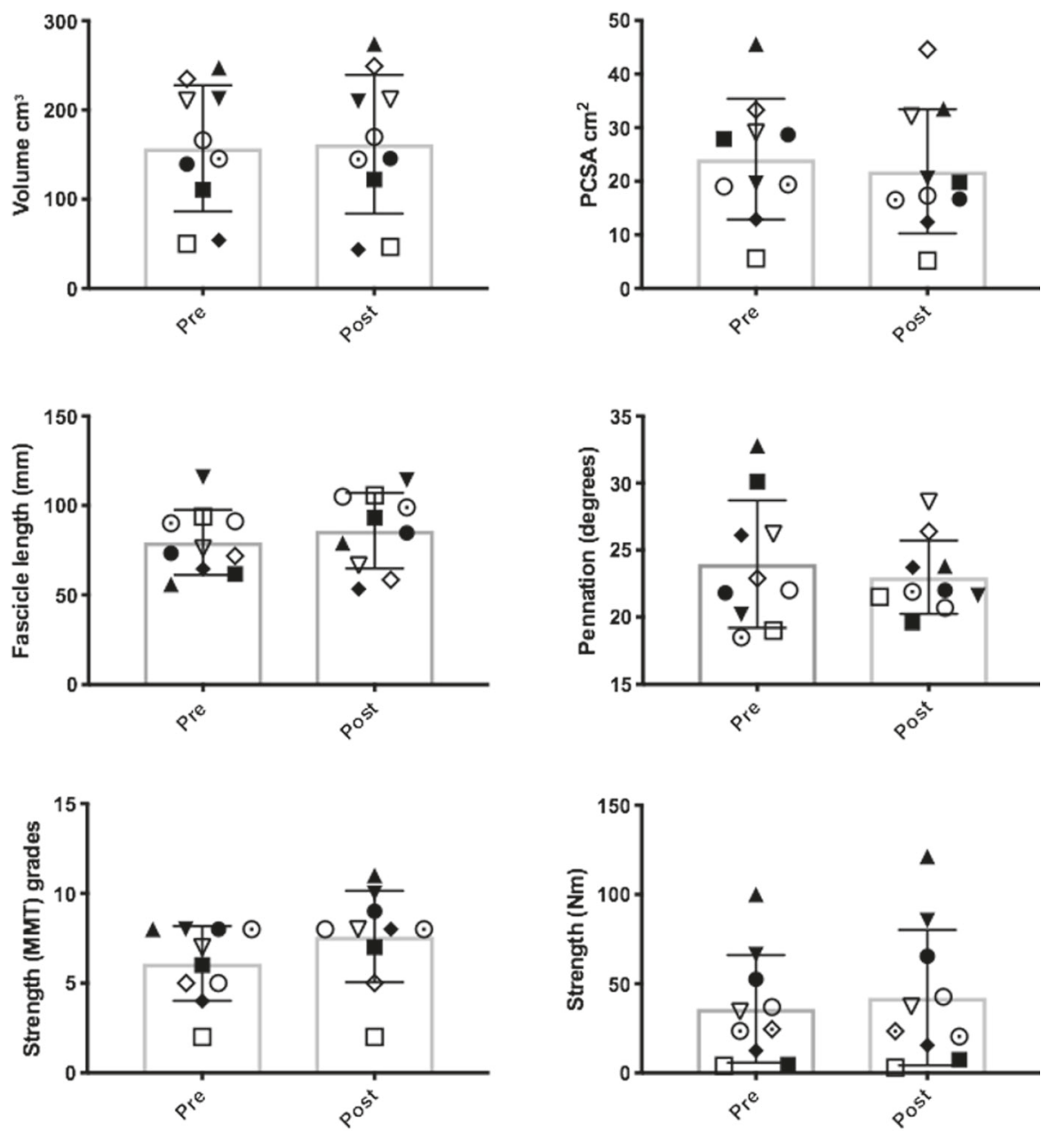

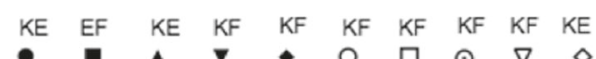

muscle architecture are typically observed only after 6 or 8 weeks of strength training. We thought that because these participants were initially weak, architectural changes may have occurred more quickly [14], but this does not seem to be the case. If we had trained our participants for longer periods, we may have observed muscle architectural changes. That does not negate the novelty of the study: no previous studies have examined the mechanisms by which voluntary strength training increases strength of partially paralysed muscles, so this finding - that strength increases produced in partially paralysed muscles by 6 weeks of training are not mediated by hypertrophy-is new.

We chose to train the participants using concentric and isometric contractions because the findings of a small cross over trial, one of the few trials of strength training in individuals with SCI, suggested isometric training produced larger increases in strength than concentric strength training [5]. However, studies of able-bodied individuals suggest that eccentric contractions lead to larger hypertrophic responses than isometric and concentric exercise [31]. Future studies could investigate whether concentric, isometric or eccentric exercise induces the most hypertrophy in people with SCI.

To our knowledge, this is the first study to use DTI to measure muscle architecture in people with SCI. It is more difficult to segment MR images of skeletal muscles from people with SCI than from able-bodied individuals, perhaps because the muscles of people with SCI may be severely atrophied and appear to contain more fat. To reduce the errors that would otherwise result from comparing pre- and post-training images, we manually segmented one of the (pre- or post-training) images for each participant and then used automated algorithms to segment the second image 
based on the first, which meant that muscle borders were segmented consistently, if not accurately.

We used DTI to measure muscle architecture because DTI allows for non-invasive study of muscle fibre architecture and is not associated with radiation risks. Unlike ultrasound imaging, DTI is able to generate measurements in three dimensions from the whole muscle [24]. However, DTI suffers from a low signal-to-noise ratio. Noise in the computed tensor fields can lead to poorly reconstructed muscle fibre fields and may cause error in measurements of muscle architecture. Our PCSA measurements are also potentially problematic as we measured PCSA by dividing volume by fascicle length at a particular joint angle rather than at optimal fascicle length. Measuring optimal fascicle length would have been ideal, but currently it is not possible, or at least would be very difficult, to measure fascicle lengths at optimal fascicle length in human muscles in vivo. Nonetheless, the MRI images allowed us to obtain good measurements of muscle volume and anatomical crosssectional area, and these measurements were not changed by 6 weeks of training. This increases our confidence in the conclusion that short-term training did not appreciably change muscle architecture.

In conclusion, we did not find any evidence that 6 weeks of strength training changed the architecture of partially paralysed muscles of people with SCI. Given the limitations of our measurements it is not possible to definitively conclude that short-term training did not increase PCSA. However, the fact that muscle volumes, which can be measured accurately from MRI, did not change strengthens the conclusion that peripheral adaptations made little or no contribution to increases in strength. Strength gains produced by 6 weeks of strength training of partially paralysed muscles of people with SCI are likely the result of improved neural drive to muscles or increases in specific muscle tension rather than changes in muscle architecture.

\section{Data availability}

Data set available in supplementary material.

Acknowledgements We thank all our participants who gave up their time to contribute to the study. We would also like to thank Fernanda Di Natal for her assistance with the study.

Funding The National Health and Medical Research Council and icare.

Author contributions $\mathrm{EAB}$ and $\mathrm{RDH}$ came up with the study concept. All authors developed the study design and protocol. EAB collected the study data. All authors, were involved in the analysis and interpretation of data. EAB prepared the first draft of the manuscript. All co-authors provided input and critical review of the manuscript leading to the final version. All authors read and approved the final manuscript.

\section{Compliance with ethical standards}

Conflict of interest The authors declare that they have no conflict of interest.

Statement of ethics We certify that all applicable institutional and governmental regulations concerning the ethical use of human volunteers were followed during the course of this research.

Publisher's note: Springer Nature remains neutral with regard to jurisdictional claims in published maps and institutional affiliations.

\section{References}

1. Thomas CK, Zaidner EY, Calancie B, Broton JG, Bigland-Ritchie BR. Muscle weakness, paralysis, and atrophy after human cervical spinal cord injury. Exp Neurol. 1997;148:414-23.

2. Beninato M, O'Kane KS, Sullivan PE. Relationship between motor FIM and muscle strength in lower cervical-level spinal cord injuries. Spinal Cord. 2004;42:533-40.

3. Bye EA, Harvey LA, Gambhir A, Kataria C, Glinsky JV, Bowden JL, et al. Strength training for partially paralysed muscles in people with recent spinal cord injury: a within-participant randomised controlled trial. Spinal Cord. 2017;55:460-5.

4. Harvey LA, Fornusek C, Bowden JL, Pontifex N, Glinsky J, Middleton JW, et al. Electrical stimulation plus progressive resistance training for leg strength in spinal cord injury: a randomized controlled trial. Spinal Cord. 2010;48:570-5.

5. Jayaraman A, Thompson CK, Rymer WZ, Hornby TG. Short-term maximal-intensity resistance training increases volitional function and strength in chronic incomplete spinal cord injury: a pilot study. J Neurol Phys Ther. 2013;37:112-7.

6. Gandevia SC. Spinal and supraspinal factors in human muscle fatigue. Physiol Rev. 2001;81:1725-89.

7. Gabriel DA, Kamen G, Frost G. Neural adaptations to resistive exercise: mechanisms and recommendations for training practices. Sports Med. 2006;36:133-49.

8. Narici MV, Hoppeler H, Kayser B, Landoni L, Claassen H, Gavardi C, et al. Human quadriceps cross-sectional area, torque and neural activation during 6 months strength training. Acta Physiol Scand. 1996;157:175-86.

9. Akima H, Takahashi H, Kuno SY, Masuda K, Masuda T, Shimojo $\mathrm{H}$, et al. Early phase adaptations of muscle use and strength to isokinetic training. Med Sci Sports Exerc. 1999;31:588-94.

10. Hickson RC, Hidaka K, Foster C, Falduto MT, Chatterton RT Jr. Successive time courses of strength development and steroid hormone responses to heavy-resistance training. J Appl Physiol. 1994;76:663-70.

11. Narici MV, Roi GS, Landoni L, Minetti AE, Cerretelli P. Changes in force, cross-sectional area and neural activation during strength training and detraining of the human quadriceps. Eur J Appl Physiol Occup Physiol. 1989;59:310-9.

12. Moritani T, deVries HA. Neural factors versus hypertrophy in the time course of muscle strength gain. Am J Phys Med. 1979;58:115-30.

13. Staron RS, Karapondo DL, Kraemer WJ, Fry AC, Gordon SE, Falkel JE, et al. Skeletal muscle adaptations during early phase of heavy-resistance training in men and women. J Appl Physiol. 1994;76:1247-55.

14. Ahtiainen JP, Pakarinen A, Alen M, Kraemer WJ, Häkkinen K. Muscle hypertrophy, hormonal adaptations and strength development during strength training in strength-trained and untrained men. Eur J Appl Physiol. 2003;89:555-63. 
15. Gorgey AS, Mather KJ, Cupp HR, Gater DR. Effects of resistance training on adiposity and metabolism after spinal cord injury. Med Sci Sports Exerc. 2012;44:165-74.

16. Lieber RL, Friden J. Functional and clinical significance of skeletal muscle architecture. Muscle Nerve. 2000;23:1647-66.

17. Fukunaga T, Roy R, Shellock F, Hodgson J, Day M, Lee P, et al. Physiological cross-sectional area of human leg muscles based on magnetic resonance imaging. J Orthop Res. 1992;10: 926-34.

18. Nordez A, Jolivet E, Sudhoff I, Bonneau D, de Guise JA, Skalli W. Comparison of methods to assess quadriceps muscle volume using magnetic resonance imaging. J Magn Reson Imaging. 2009;30:1116-23.

19. Nijholt W, Scafoglieri A, Jager-Wittenaar H, Hobbelen JSM, van der Schans CP. The reliability and validity of ultrasound to quantify muscles in older adults: a systematic review. J Cachex Sarcopenia Muscle. 2017;8:702-12.

20. McDonald ML, Diaz AA, Ross JC, San Jose Estepar R, Zhou L, Regan EA, et al. Quantitative computed tomography measures of pectoralis muscle area and disease severity in chronic obstructive pulmonary disease. A cross-sectional study. Ann Am Thorac Soc. 2014;11:326-34.

21. Kwah LK, Pinto RZ, Diong J, Herbert RD. Reliability and validity of ultrasound measurements of muscle fascicle length and pennation in humans: a systematic review. J Appl Physiol. 2013;114:761-9.

22. Bolsterlee B, Gandevia SC, Herbert RD. Effect of transducer orientation on errors in ultrasound image-based measurements of human medial gastrocnemius muscle fascicle length and pennation. PLoS ONE. 2016;11:e0157273.

23. Bolsterlee B, Gandevia SC, Herbert RD. Ultrasound imaging of the human medial gastrocnemius muscle: how to orient the transducer so that muscle fascicles lie in the image plane. J Biomech. 2016;49:1002-8.

24. Bolsterlee B, Veeger HE, van der Helm FC, Gandevia SC, Herbert RD. Comparison of measurements of medial gastrocnemius architectural parameters from ultrasound and diffusion tensor images. J Biomech. 2015;48:1133-40.

25. Damon BM, Froeling M, Buck AK, Oudeman J, Ding Z, Nederveen AJ, et al. Skeletal muscle diffusion tensor-MRI fiber tracking: rationale, data acquisition and analysis methods, applications and future directions. NMR Biomed. 2017;30:3.

26. Bolsterlee B, D'Souza A, Gandevia SC, Herbert RD. How does passive lengthening change the architecture of the human medial gastrocnemius muscle? J Appl Physiol. 2017;122:727-38.

27. Paternostro-Sluga T, Grim-Stieger M, Posch M, Schuhfried O, Vacariu G, Mittermaier C, et al. Reliability and validity of the Medical Research Council (MRC) scale and a modified scale for testing muscle strength in patients with radial palsy. J Rehabil Med. 2008;40:665-71.

28. Klein S, Staring M, Murphy K, Viergever MA, Pluim JP. elastix: a toolbox for intensity-based medical image registration. IEEE Trans Med Imaging. 2010;29:196-205.

29. Shamonin DP, Bron EE, Lelieveldt BP, Smits M, Klein S, Staring $\mathrm{M}$. Fast parallel image registration on $\mathrm{CPU}$ and GPU for diagnostic classification of Alzheimer's disease. Front Neuroinform. 2013;7:50.

30. Gregory CM, Bowden MG, Jayaraman A, Shah P, Behrman A, Kautz SA, et al. Resistance training and locomotor recovery after incomplete spinal cord injury: a case series. Spinal Cord. 2007:45:522-30.

31. Farthing JP, Chilibeck PD. The effects of eccentric and concentric training at different velocities on muscle hypertrophy. Eur J Appl Physiol. 2003;89:578-86. 